\title{
El acoso sexual callejero hacia las mujeres del mercado Bahía Río de Portoviejo
}

\author{
Street sexual harassment of women in the Bahía Río market in Portoviejo \\ Assédio sexual de rua a mulheres no mercado Bahía Río em Portoviejo \\ Saby Daniela Moreira Alvarez \\ sabydanielapsicl@gmail.com \\ https://orcid.org/0000-0001-6746-0341 \\ Adriana Josselyn Zambrano Valderrama \\ joadri777@gmail.com \\ https://orcid.org/0000-0001-9984-9722
}

Universidad Técnica de Manabí, Portoviejo, Ecuador

Universidad Técnica de Manabí, Portoviejo, Ecuador

Recibido: febrero 2021 / Revisado: marzo 2021 / Aceptado: abril 2021 / Publicado: mayo 2021

\section{RESUMEN}

La investigación tuvo como objetivo describir el fenómeno del acoso sexual callejero hacia las mujeres desde la perspectiva de los vendedores del mercado Bahía Río de Portoviejo, desde Noviembre 2020 hasta Febrero 2021. La concepción teórica se apoyó fundamentalmente en los autores Restrepo (2017) Expresioners (2019) Farez (2019) Cruz (2017) y Gaytan (2009) entre otros. Se diseñó una metodología de tipo cuanticualitativa para indagar la forma en que se manifiesta el fenómeno estudiado. Por tal motivo se aplicó una encuesta con preguntas abiertas y cerradas a grupos focales, para el análisis de las desigualdades sociales sustentadas en temas de género. El masculino es consciente de que el acoso sexual callejero es un tipo de violencia que, al igual que los otros, genera repercusiones en sus víctimas. Y que a pesar de ser un suceso social y culturalmente naturalizado, normalizado o invisibilizado, se considera un problema porque no permite a la mujer, disfrutar del derecho al libre tránsito y estadía por los espacios públicos.

Palabras clave: Acoso sexual callejero; mercado; género; desigualdad
ABSTRACT

RESUMO

The objective of the research was to describe the phenomenon of street sexual harassment towards women from the perspective of the vendors of the Bahía Río de Portoviejo market, from November 2020 to February 2021. The theoretical conception was based mainly on the authors Restrepo (2017) Expresioners ( 2019) Farez (2019) Cruz (2017) and Gaytan (2009) among others. A quantitativequalitative methodology was designed to investigate the way in which the studied phenomenon manifests itself. For this reason, a survey with open and closed questions was applied to focus groups, for the analysis of social inequalities based on gender issues. The male is aware that street sexual harassment is a type of violence that, like the others, generates repercussions on its victims. And that despite being a social and culturally naturalized, normalized or invisible event, it is considered a problem because it does not allow women to enjoy the right to free movement and stay in public spaces.

Key words:

Street sexual harassment; market; gender; inequality
O objetivo da pesquisa foi descrever - fenômeno do assédio sexual de rua a mulheres sob a ótica dos vendedores ambulantes no mercado da Bahia Río de Portoviejo, de novembro de 2020 a fevereiro de 2021. A concepção teórica foi apoiada fundamentalmente pelos autores Restrepo(2017) Expresioners (2019) Farez (2019) Cruz (2017) e Gaytan (2009) entre outros. Uma metodologia quantitativoqualitativa foi desenhada para investigar a forma como o fenômeno estudado se manifesta. Por esse motivo, foi aplicada uma pesquisa com questões abertas e fechadas a grupos focais, para análise das desigualdades sociais a partir das questões de gênero. $O$ homem tem consciência de que o assédio sexual nas ruas é uma forma de violência que, como as demais, repercute nas suas vítimas. E que apesar de ser um acontecimento social e culturalmente naturalizado, normalizado ou invisível, é considerado problemático porque não permite que as mulheres usufruam do direito à livre circulação e permanência nos espaços públicos.

Palavras-chave:

Assédio sexual de rua; mercado; Gênero sexual; desigualdade 


\section{INTRODUCCIÓN}

Estudios relacionados con el tema violencia de género en cuanto al acoso sexual fueron realizados en la Comisión Económica para América Latina y el Caribe (Cepal) Allí se analizan encuestas públicas del OCAC y el Sernam. Ambas encuestas realizadas en Chile, México, Perú, Colombia, Argentina y Paraguay, coinciden que 6 de cada 10 mujeres sufren con frecuencia de acoso sexual en espacios públicos. Un tercio de estas encuestadas afirman que han sido víctimas de acercamientos más íntimos y agresivos, más comúnmente en transportes públicos o sitios de mucha conglomeración (Cepal, 2015. Citado por Restrepo, 2017, p.35).

Aun cuando se trata de evidenciar el problema de acoso callejero por medio de encuestas latinoamericanas, fue necesario centrarse en cifras que demostraron que el problema existe y que sobre todo afecta a la población ecuatoriana.

Un estudio realizado por el Instituto Nacional de Estadística y Censos [INEC] reveló que 65 de cada 100 mujeres en el Ecuador, han experimentado por lo menos un hecho de algún tipo de violencia a lo largo de su vida, siendo la agresión psicológica la segunda de la lista. A diario, miles de mujeres son acosadas en las calles, mientras van al mercado o caminan por las aceras. Reciben miradas, chiflidos o palabras que para la sociedad son normales, porque se las ha visto como un cumplido y solo queda aceptarlos; aunque en realidad sean un tipo de violencia (Expresionesr, 2019, pp.6-7) Al respecto opina Farez:

El acoso sexual callejero es una forma de violencia estructural presente en la sociedad, por la vigencia de una jerarquización en los roles asignados a hombres y mujeres; dicha violencia va en perjuicio de las posibilidades de uso del espacio público para el género femenino. (2019, p. 2)
Sin duda, es una forma de violencia que frecuentemente -casi a diario- sufren millones de mujeres al constituirse como blancos vulnerables de insinuaciones sexuales en lugares públicos. En la práctica, ocurre que la mayoría de las féminas que han sido víctimas del acoso sexual callejero no saben cómo reaccionar o defenderse. Esto incentiva conductas de olvido puesto que no hacen nada y, asimismo, comportamientos asociados con el silencio. Estas simples acciones vulneran sus derechos, puesto que toda fémina tiene derecho a una vida libre de violencia, tanto en el ámbito público como privado.

Al observar este panorama, las investigadoras asumieron y determinaron las siguientes formulaciones: ¿Cuáles son las características del acoso sexual callejero desde la perspectiva de los vendedores del mercado Bahía Río de Portoviejo, desde Noviembre 2020 hasta Febrero 2021? ¿Cuáles son los tipos de acoso sexual callejero más comunes? ¿Cuáles son los motivos que inducen la práctica del acoso sexual callejero hacia las mujeres? ¿Cuál es la opinión de los vendedores sobre los pensamientos de los hombres que realizan acoso sexual callejero? y ¿qué piensan los vendedores sobre los sentimientos de los hombres que realizan acoso sexual callejero?

Por consiguientes se redactaron los objetivos siguientes:

Describir el fenómeno del acoso sexual callejero hacia las mujeres desde la perspectiva de los vendedores del mercado Bahía Río de Portoviejo, desde noviembre 2020 hasta febrero 2021. Seguidamente, se categorizan los tipos de acoso sexual callejero más comunes, luego se identifica los motivos que inducen la práctica del acoso sexual callejero hacia las mujeres, para después indagar la opinión de los vendedores sobre los pensamientos de los hombres que realizan acoso sexual callejero, en donde se 
finaliza con los pensamientos de los vendedores sobre los sentimientos de los hombres que realizan acoso sexual callejero.

La investigación buscó sustentar teóricamente las principales variables que conforman el tema. En primer lugar el acoso sexual callejero como una forma de violencia de género $y$, en segundo término, la perspectiva subjetiva que tiene una población específica sobre dicho fenómeno. En este caso, la de los vendedores del mercado Bahía Río de Portoviejo.

No es secreto afirmar que se han efectuado estudios sobre la violencia que sufren las personas del género femenino en el ámbito de las dinámicas familiares o de lo doméstico. Sin embargo, en esta tesis se abordó el acoso sexual callejero ejercido en lugares públicos, para denotar la discriminación de género que está estructuralmente presente no solo en las familias, sino en todas las dinámicas sociales. La cantidad de estudios académicos que se han realizado permite un amplio acceso y facilidad del estudio bibliográfico del fenómeno.

De esta manera se afirmó que el acoso sexual callejero se ha convertido en un problema de gran relevancia a pesar de estar invisibilizado. Al profundizar este fenómeno es necesario abordar el tema que se vincula con la garantía de derechos de más de la mitad de la población nacional: las mujeres. Es común que las indagaciones académicas solo se centren en la población femenina y olvidan e ignoran las opiniones del género masculino. Incluso, son muy pocos los autores que consideran a ambos géneros y casi nulos los que buscan conocer la perspectiva solamente del género masculino.

De ahí resultó interesante e innovador el deseo de indagar acerca de las percepciones de los hombres y de los principales perpetradores de los actos de acoso sexual en espacios públicos -que usualmente son causados por parte del género masculino-. De esta forma se abordaron diversos factores, por ejemplo: tipos de acoso más comunes, posibles motivaciones, pensamientos y sentimientos por la acometida de dichos actos. Sin duda, esto permite conocer la presencia o ausencia de una conciencia por parte del género masculino sobre la gravedad del acoso como fenómeno social.

Una de las principales motivaciones para el desarrollo de este estudio se fundamentó en evidenciar la existencia de dificultades en otros estudios realizados, para identificar una metodología sistemática que aborde la percepción que tienen grupos sociales específicos (por ejemplo, los hombres) sobre el fenómeno del acoso sexual callejero. Por esta razón se optó por emplear un enfoque de tipo cuanti-cualitativo o mixto, con la finalidad de recolectar, analizar y vincular los datos obtenidos en la investigación.

\section{MÉTODO}

El tipo de estudio desarrollado fue el descriptivo y tuvo como finalidad abordar los objetivos planteados mediante situaciones y eventos. Buscó especificar las propiedades importantes en personas, grupos, comuni $\neg$ dades o cualquier otro fenómeno que sea sometido a análisis (Hernández, 1997, p. 43)

Esta tesis diseñó una metodología de tipo cuanti-cualitativa para indagar la forma en que se manifiesta el acoso sexual callejero. Por tal motivo se aplicó una encuesta con preguntas abiertas y cerradas a grupos focales, para el análisis de las desigualdades sociales sustentadas en temas de género. La muestra de estudio escogida abarcó a 108 vendedores del mercado de Bahía Río de Portoviejo. 
Fundamentalmente se empleó como referencia el cuestionario realizado por una estudiante de la Universidad Autónoma de Barcelona, quien utilizó como guía el estudio nacional sobre el acoso sexual y asalto ejecutado por la Asociación Stop Street Harassment (2014) Y la encuesta realizada por una estudiante de la Universidad de Cuenca, quien se basó en el cuestionario utilizado en una investigación sobre el acoso sexual realizado en la ciudad de Cuenca en el año 2015.

\section{RESULTADOS}

Las ciento ocho encuestas aplicadas permitieron identificar las conductas, los tipos de acoso sexual callejero más comunes, las motivaciones, los pensamientos y los sentimientos, que desde la perspectiva de los encuestados experimentan los hombres cuando realizan esas conductas.

Seguidamente, a manera de ejemplo, se desglosan algunas preguntas representativas con sus respectivas tablas, gráficas y análisis.

1.- ¿Qué es acoso sexual callejero?

Tabla 1. Datos de la muestra escogida (1 pregunta).

\section{Opciones}

Frecuencia

Porcentaje

Es la naturalización y normalización de los piropos realizados en las calles como galantería que resalta la belleza femenina.

16

$15 \%$

Es una experiencia que debe ser aceptada por las mujeres, en la que uno o varios hombres se dirigen hacia ellas usando expresiones verbales o no verbales con contenido sexual.

Es una práctica de violencia física, psicológica y sexual acompañada de interacciones indeseadas en espacios públicos y entre desconocidos. Que son motivadas por el género efectivo o percibido, orientación sexual o expresión de género de una persona y que hacen que la víctima se sienta molesta, enojada, humillada o espantada.

Es aceptar que cuando una mujer transita por los espacios públicos, pasa a ser un objeto de insinuaciones mediante expresiones de contenido sexual.
$4 \quad 4 \%$

81

$75 \%$

7

$6 \%$

673

$100 \%$

Total

Fuente: elaboración propia basada en datos de los vendedores del Mercado Bahía Río de Portoviejo. 


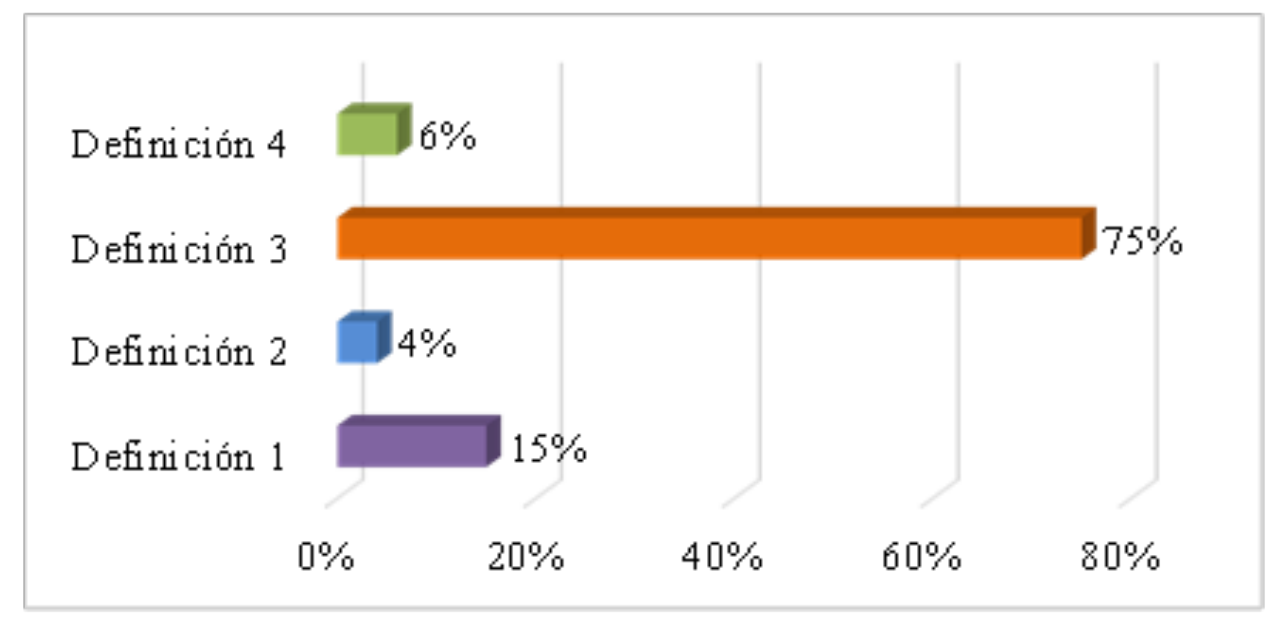

Figura 1. Datos de la muestra escogida ( $1^{\text {a }}$ pregunta). Fuente: elaboración propia basada en datos de los vendedores del Mercado Bahía Río de Portoviejo.

\section{Análisis e interpretación}

En esta pregunta, 81 vendedores (75\%) establecieron como acoso sexual callejero la definición de la organización estadounidense Stop Street Harassment (2014).

Es una práctica de violencia física, psicológica y sexual acompañada de interacciones indeseadas en espacios públicos entre desconocidos que son motivadas por el género efectivo o percibido, orientación sexual o expresión de género de una persona y que hacen que la víctima se sienta molesta, enojada, humillada, o espantada. (párr. 13).

Por otro lado, 16 vendedores (15\%) afirmaron que el acoso sexual callejero es la naturalización y normalización de los piropos realizados en las calles como galantería, en el que se resalta la belleza femenina. En contraste, 7 de los participantes (6\%) eligieron la definición cuatro, la cual plantea que el acoso sexual callejero es aceptar que cuando una mujer transita por los espacios públicos pasa a ser un objeto de insinuaciones mediante expresiones de contenido sexual. Y, 4 de ellos (4\%) pensaron que el acoso sexual es una experiencia que debe ser aceptada por las féminas, en la que uno o varios hombres se dirigen hacia ellas con términos verbales o no verbales con contenido sexual.

Es preciso destacar otra interrogante ¿Cuáles de las siguientes opciones son considerados acoso sexual callejero?

Al igual que la pregunta anterior, en la Tabla 2 , se expresa como en esta interrogante los encuestados podían elegir más de una opción. Gráfico y Tabla 1. 
Tabla 2. Datos de la muestra escogida (4 pregunta).

\begin{tabular}{lcc}
\hline \multicolumn{1}{c}{ Opciones } & Frecuencia & Porcentaje \\
\hline Piropos suaves (halagos) & $31 / 108$ & $4,6 \%$ \\
Gestos obscenos & $56 / 108$ & $8,3 \%$ \\
Fotografías y grabaciones del cuerpo, no consentidas y con connotación sexual & $76 / 108$ & $11,3 \%$ \\
Silbidos y otros sonidos (besos, bocinazos) & $42 / 108$ & $6,3 \%$ \\
Piropos agresivos (alusiones al cuerpo y al acto sexual) & $76 / 108$ & $11,3 \%$ \\
Persecución (a pie o por medio de un transporte) & $70 / 108$ & $10,4 \%$ \\
Tocar, agarrar o rozar de manera intencional a una desconocida & $91 / 108$ & $13,5 \%$ \\
Enseñar los genitales a una desconocida en la calle & $82 / 108$ & $12,2 \%$ \\
Masturbarse en la calle mirando a una desconocida & $77 / 108$ & $11,4 \%$ \\
Miradas con intenciones sexuales & $72 / 108$ & $10,7 \%$ \\
Total & $\mathbf{6 7 3}$ & $\mathbf{1 0 0} \%$ \\
\hline
\end{tabular}

Fuente: elaboración propia basada en datos de los vendedores del Mercado Bahía Río de Portoviejo.

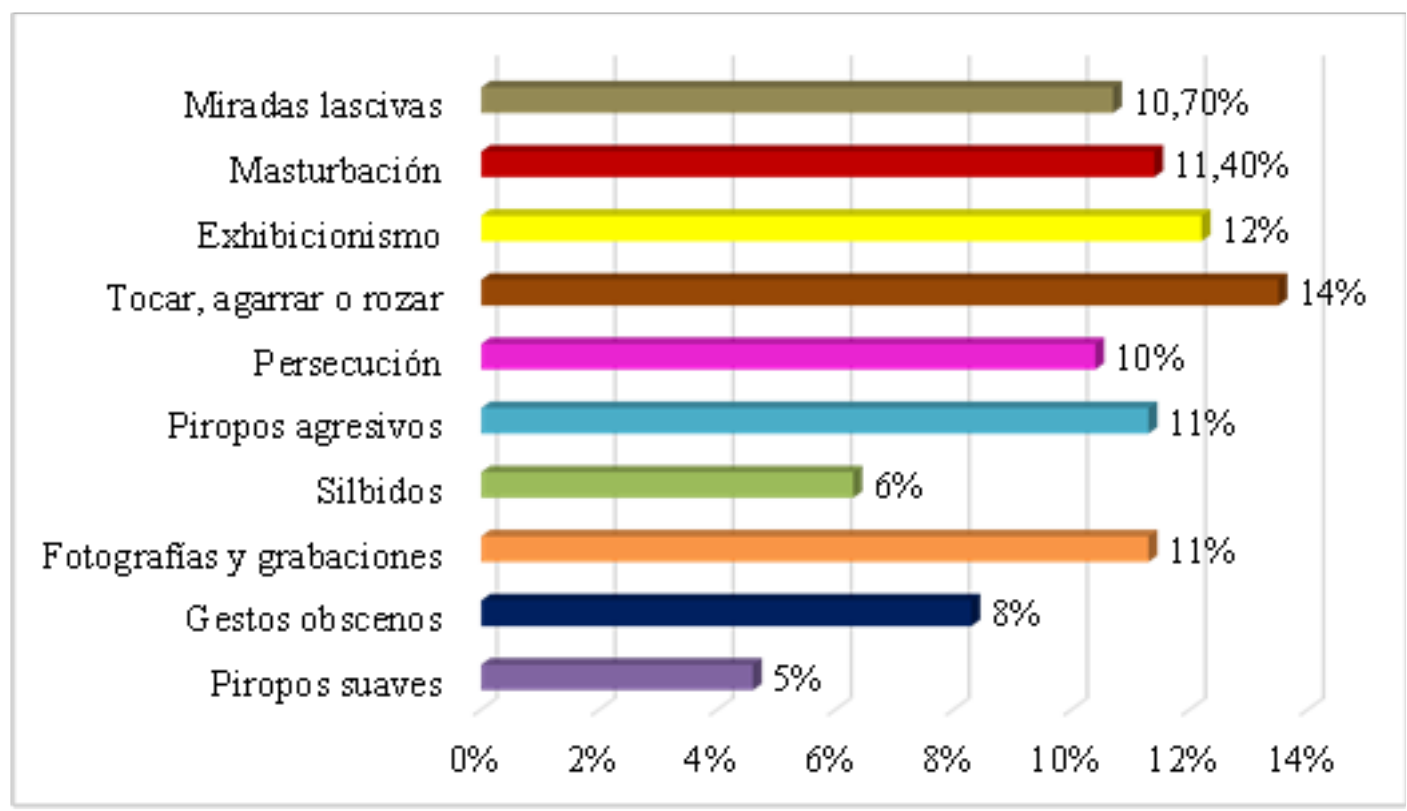

Figura 2. Datos de la muestra escogida. (Fuente: elaboración propia basada en datos de los vendedores del Mercado Bahía Río de Portoviejo).

\section{Análisis e interpretación}

Al igual que la pregunta anterior, en esta interrogante los encuestados podían elegir más de una opción. Los tipos de acoso sexual callejero se obtuvieron de la clasificación planteada por
Gaytán (2009) en su estudio realizado en Grounded Theory, clasifica las formas de acoso callejero en: Acoso expresivo entendido como una interacción violenta y subjetiva, que incluye miradas lascivas, expresiones faciales, corporales entre otras; acoso 
verbal como violencia expresada a través de la comunicación, incluyen piropos y expresiones verbales de tipo sexual; persecuciones cuando el acosador mantiene una cercanía física insistente con su víctima en los espacios públicos; acoso físico siendo esta una de las agresiones más graves e intencionales, el violentador toca el cuerpo de la víctima como nalgas, caderas, órganos sexuales y senos, sin su autorización en un lugar público y el exhibicionismo que consiste en la exposición de los genitales a una o varias víctimas, pudiendo ir acompañada de masturbación y de eyaculación.

Uno de los comportamientos más considerado como acoso sexual callejero fue el de tocar, agarrar o rozar, dado que 91 de los 108 encuestados $(13,5 \%)$ lo seleccionaron. Otra de las conductas mayormente seleccionada fue la de enseñar los genitales a una desconocida en la calle $(12,2 \%)$ que fue seleccionada por 82 vendedores. En tercer lugar está el masturbarse en la calle mirando a una desconocida, la cual fue elegida por 77 vendedores $(11,4 \%)$ Las fotografías y grabaciones del cuerpo no consentidas y con connotación sexual, al igual que los piropos agresivos, fueron escogidas por 76 vendedores (11,3\%) Por otro lado, 72 participantes $(10,7 \%)$ optaron por las miradas con intenciones sexuales del total de alternativas elegidas. En contraste, 70 de los involucrados $(10,4 \%)$ se inclinaron por las persecuciones. Los gestos obscenos representaron el 8,3\%, lo cual significa que fueron seleccionadas 56 veces. Los silbidos y otros sonidos representaron el 6,3\% (42 adeptos) y, por su parte, los piropos suaves con un $4,6 \%$ del total de 21 elecciones.

Comparando con la investigación realizada por Farez (2019) las acciones de acoso callejero realizadas en espacios públicos, en relación al contacto corporal el $64,2 \%$ de los encuestados reveló haber tocado la cintura y hablado al oído de la víctima, el $14,7 \%$ indicó haber realizado agarrones de senos, vulva o trasero. El acercamiento intimidante fue utilizado por el 10,3\%., el $1 \%$ reportó haber violado a la víctima ( 2 casos) el exhibicionismo o masturbación fue practicado por el $8,3 \%$, la persecución a pie o en medio de transporte fue reportada por el 5,9\% y los silbidos, besos, jadeos, bocinazos, miradas lascivas, representaron el $70,1 \%$.

Los resultados obtenidos también concuerdan con la investigación de Villa ejecutada en Perú, en donde: La mayoría de los hombres $(61,6 \%)$ creen que si una mujer recibe un piropo de un desconocido debería sentirse halagada y el $41,1 \%$ cree que mientras no toque a una mujer desconocida, lanzarle piropos o mirarla de manera persistente está permitido (Instituto de Opinión Pública de la PUCP, como se citó en Cruz, 2017, p. 21).

Se suma al proceso investigativo la siguiente cuestión ¿cuáles usted considera son pensamientos que tienen los hombres que realizan acoso sexual callejero?

Se devela en la Tabla 3 la relevancia de las expresiones que se apoyan en lo estético como acoso. 
Tabla 3. Datos de la muestra escogida ( $9^{\mathrm{a}}$ pregunta).

\begin{tabular}{lcc}
\hline \multicolumn{1}{c}{ Opciones } & Frecuencia & Porcentaje \\
\hline "A ella le gusta" & $22 / 108$ & $9,5 \%$ \\
"Soy un hombre puedo decir o hacer lo que quiera" & $46 / 108$ & $18,9 \%$ \\
"Ella lo está buscando por cómo se viste" & $59 / 108$ & $24,3 \%$ \\
"No importa que le diga o haga ella no hará nada" & $23 / 108$ & $9,5 \%$ \\
"Le estoy haciendo sentir bonita" & $17 / 108$ & $7 \%$ \\
"Me gusta" & $24 / 108$ & $9,9 \%$ \\
"Soy un galán" & $18 / 108$ & $7,4 \%$ \\
"La belleza de la mujer debe ser resaltada" & $33 / 108$ & $13,6 \%$ \\
"Son solo palabras, no le afectan" & $1 / 108$ & $0,4 \%$ \\
Total & $\mathbf{2 4 3}$ & $\mathbf{1 0 0} \%$ \\
\hline
\end{tabular}

Fuente: elaboración propia basada en datos de los vendedores del Mercado Bahía Río de Portoviejo.

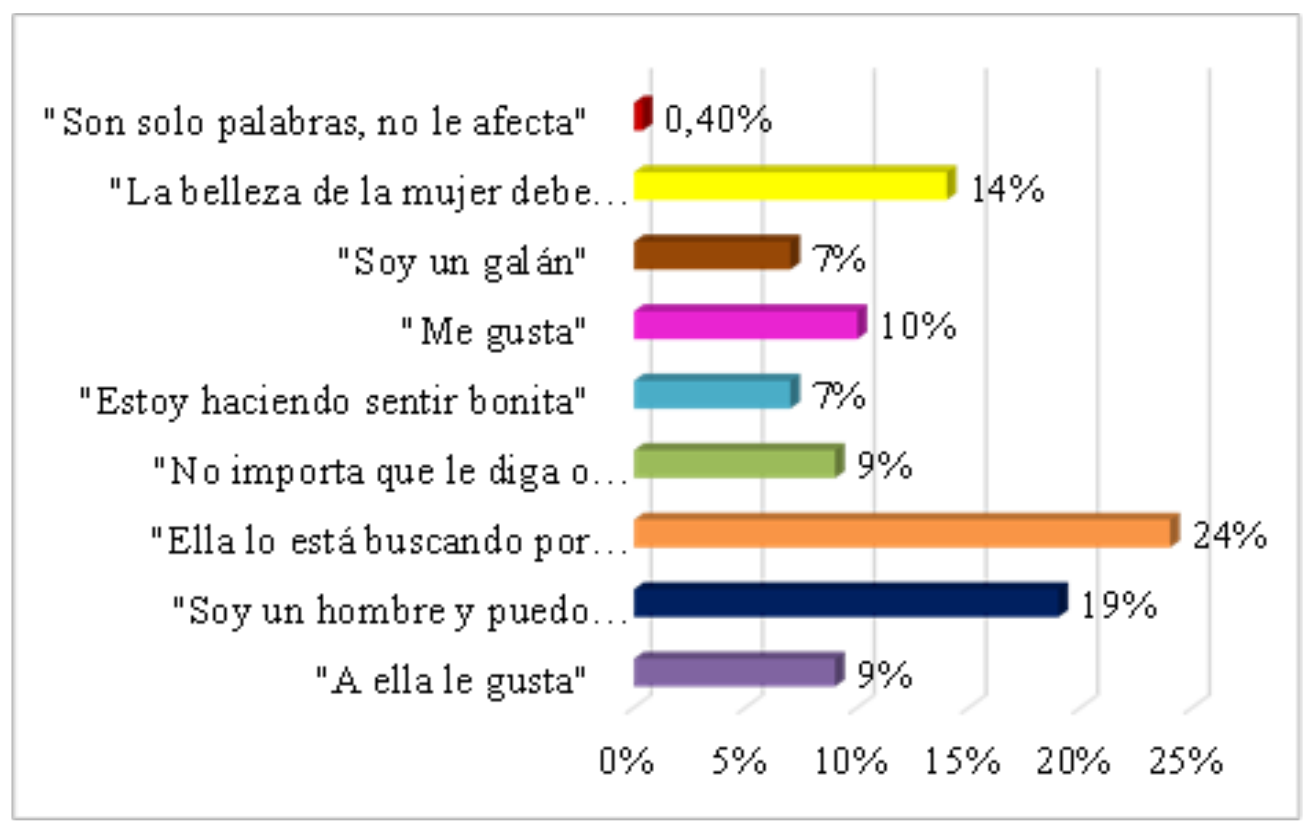

Figura 3. Datos de la muestra escogida. Fuente: elaboración propia basada en datos de los vendedores del Mercado Bahía Río de Portoviejo.

\section{Análisis e interpretación}

En esta pregunta los vendedores consideraron que el pensamiento más recurrente en hombres que realizan acoso sexual callejero es: "ella lo está buscando por cómo se viste" con 59 selecciones (24\% del total de respuestas seleccionadas) Con el 19\% (46 veces elegido) está el pensamiento "soy un hombre y puedo decir o hacer lo que quiera"; "la belleza de la mujer debe ser resaltada" representó el $14 \%$ del total de respuestas elegidas (33) Posteriormente, está la alternativa "me gusta" (10\%- 24 veces) "no importa que le diga o haga ella no hará nada" ( $9 \%-23$ veces) y "a ella le gusta" ( $9 \%$ - 22 veces) Además, con un 7\% están las opciones: 
"soy un galán" y "le estoy haciendo sentir bonita"; la primera fue escogida por 18 vendedores y la segunda por 17. Por último, 1 solo participante indicó que los hombres que realizan acoso sexual callejero de seguro piensan que "son solo palabras, no le afectan".

Esta información concuerda con la creencia generalizada por parte de los varones (cerca del $60 \%$ quienes consideran que el modo de vestir de una mujer es lo que provoca o justifica la existencia del acoso en las calles (Instituto de género y promoción de la igualdad, 2016, como se citó en Farez, 2019, p.29).

Por otra parte se añade ¿cuáles usted considera son sentimientos que experimentan los hombres que realizan acoso sexual callejero?
En este cuestionamiento, 57 de los 108 encuestados señalaron que los hombres que efectúan acoso sexual callejero sienten superioridad; cifra que representa el $33 \%$ de alternativas seleccionadas. En segundo lugar está la satisfacción que fue escogida 48 veces (28\%) Le sigue la inferioridad seleccionada 20 veces que sería el $12 \%$; luego está la euforia con un $10 \%$, puesto que fue elegida por 17 vendedores. De forma muy cercana, está la admiración con un 9 \% seleccionada por 15 encuestados. Los demás resultados se resumen de la siguiente manera: culpa (11 veces- $6 \%$ ) y el deseo con un $2 \%$ marcado por 4 vendedores.

Tabla 4. Datos de la muestra escogida ( $11^{\text {a }}$ pregunta).

\begin{tabular}{|c|c|c|}
\hline Opciones & Frecuencia & Porcentaje \\
\hline Admiración & $15 / 108$ & $8,7 \%$ \\
\hline Euforia & $17 / 108$ & $9,9 \%$ \\
\hline Satisfacción & $48 / 108$ & $27,9 \%$ \\
\hline Culpa & $11 / 108$ & $6,4 \%$ \\
\hline Superioridad & $57 / 108$ & $33,1 \%$ \\
\hline Inferioridad & $20 / 108$ & $11,6 \%$ \\
\hline Deseo & 4/ 108 & $2,3 \%$ \\
\hline Total & 172 & $100 \%$ \\
\hline
\end{tabular}

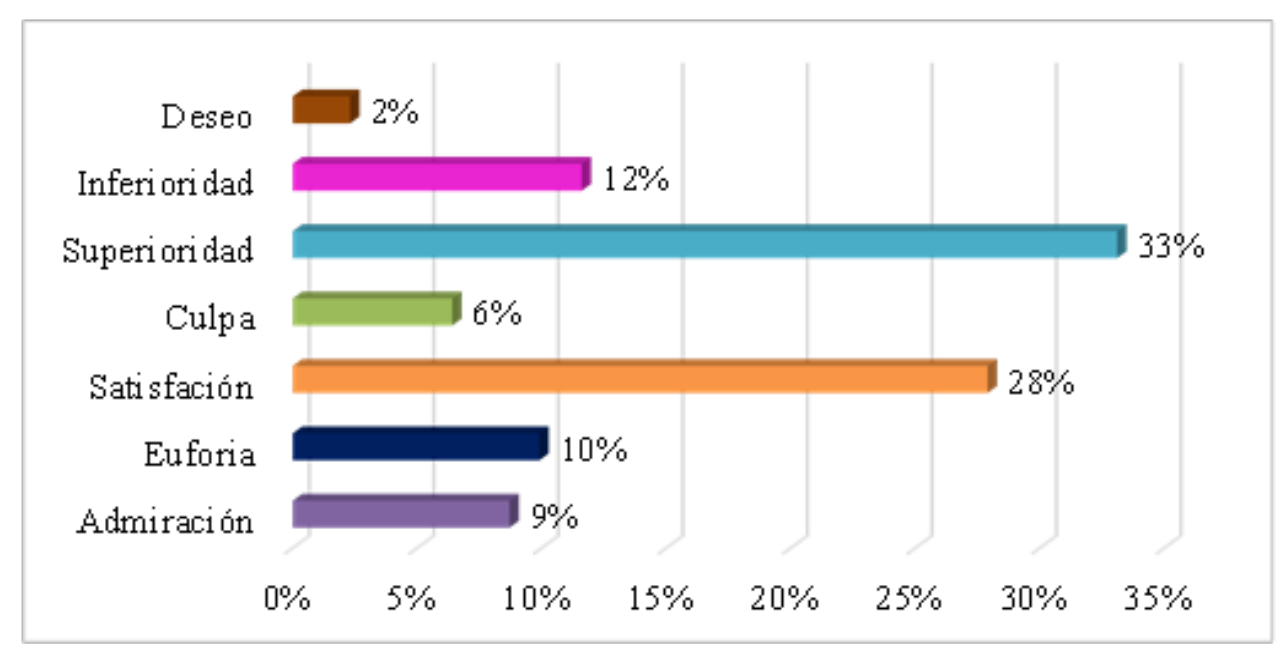

Figura 4. Datos de la muestra escogida (Fuente: elaboración propia basada en datos de los vendedores del Mercado Bahía Río de Portoviejo). 


\section{Análisis e interpretación}

La información recaudada concuerda con la investigación de Rodríguez (2020) en donde se establece que el acoso sexual callejero, a través de cualquiera de sus formas lo que busca es reforzar la masculinidad. El objetivo de este tipo de comportamientos no es tanto el agredir a la mujer sino reforzar la propia masculinidad y con ello lograr un sentimiento de superioridad.

En este sentido, es oportuno destacar los alcances de objetivos y resultados. En primera instancia la investigación se llevó a cabo con la finalidad de describir el fenómeno del acoso sexual callejero hacia las mujeres desde la perspectiva de los vendedores del mercado Bahía Río de Portoviejo. El primer específico permitió categorizar los tipos de acoso sexual callejero más comunes, identificados como silbidos $u$ otros sonidos, piropos suaves y piropos agresivos. El segundo objetivo específico buscó identificar los motivos que inducen la práctica del acoso sexual callejero hacia las féminas, entre los cuales se encuentran: para quedar bien frente a otros hombres; por la vestimenta de la mujer; por demostrar su masculinidad y por la atracción sexual que se siente hacia la víctima.

El tercer objetivo indaga los diversos pensamientos de la mayoría de los acosadores. Comparten que es la mujer quien busca que la acosen por su manera de vestir, incluso, el sujeto puede hacer y decir lo que quiera, puesto que se debe resaltar la belleza del género contrario.

Por último, el objetivo cuatro hizo referencia a los pensamientos de los vendedores sobre los sentimientos de los hombres que realizan acoso sexual callejero. Según el análisis existió un completo desacuerdo con los sentimientos de superioridad, satisfacción e inferioridad experimentados por los individuos que realizan dicha práctica.
No es posible negar hoy día que a pesar de que el acoso sexual callejero es una práctica normalizada y naturalizada por gran parte de la sociedad, existe una invisibilización sobre las consecuencias que esta problemática produce en el género femenino.

\section{CONCLUSIONES}

La mayoría de los vendedores del mercado Bahía Río de Portoviejo, desde Noviembre 2020 hasta Febrero 2021, identificaron como adecuada la definición de la organización estadounidense Stop Street Harassment (2014) cuando señala que el género masculino es consciente de que el acoso sexual callejero es un tipo de violencia que, al igual que los otros, genera repercusiones en sus víctimas. Y que, a pesar de ser un fenómeno naturalizado, normalizado o invisibilizado, lo consideran como un problema.

Los comportamientos de acoso sexual callejero más observados por los participantes a lo largo de toda su vida son: silbidos, piropos suaves y piropos agresivos. Muchos de los vendedores no identificaban los piropos suaves como uno de esta categoría, puesto que estas conductas no generan ninguna molestia para las mujeres. Por el contrario lo concerniente a un acoso físico como el tocar, rozar o agarrar a una desconocida sin su consentimiento fue el más señalado.

Desde el punto de vista de los encuestados los hombres realizan acoso sexual callejero para quedar bien frente a sus pares. Prueba de ello es que este tipo de situaciones ocurren cuando están acompañados. Otro de los motivos seleccionados fue por la vestimenta de la mujer, puesto que piensan que si se viste provocativamente, ella está propensa e inclusive incita para que otros le falten el respeto. 
También está la opción de demostrar su masculinidad, lo cual va de la mano con la cultura manabita, quien establece los comportamientos y actitudes que debe tener un hombre. Existe una creencia altamente machista, lo cual fue un aspecto mencionado por la mayoría de ellos al considerar a la cultura como un aspecto que motiva el acoso sexual callejero.

Los pensamientos que cursan por la mente de los agresores fueron: "ella se lo busca por cómo se viste", "soy un hombre y puedo hacer/decir lo que quiera" y "la belleza de la mujer debe ser resaltada". En lo que respecta a los pensamientos de los vendedores acerca de los sentimientos de los acosadores, los mismos desaprueban los sentimientos de superioridad, satisfacción e inferioridad experimentados por los agresores.

\section{REFERENCIAS}

Cruz, D. (2017). Propiedades psicométricas de una escala de acoso sexual callejero en estudiantes mujeres de una universidad privada de la ciudad de Piura, 2017. Universidad César Vallejo. https://repositorio.ucv.edu.pe/ bitstream/handle/20.500.12692/10630/cruz pd.pdf?sequence $=1$ \&isAllowed $=y$

Farez, Y. (2019). El acoso sexual callejero desde la perspectiva de los varones estudiantes de la Universidad de Cuenca. Universidad de Cuencia. http://dspace.ucuenca.edu.ec/ bitstream/123456789/32125/1/trabajo\%20 de\%20titulacion.\%20pdf.pdf

Fernández, M. (2016). Percepción de los varones jóvenes acerca del Acoso Sexual Callejero dirigido a las mujeres. Universidad de la República Uruguay. https://sifp.psico.edu.uy/ sites/default/files/Trabajos\%20finales/\%20 Archivos/tfg-magali_fernandez.pdf

Gaytan, P. (2009). El acoso sexual en lugares públicos: un estudio desde la Grounded Theory. El cotidiano, 22(143), 5-17. https://www.redalyc. org/pdf/325/32514302.pdf

Hernández, R., Fernández, C., \& Baptista, P. (1997). Metodología de la Investigación. México: McGraw Hill

Restrepo, C. (2017). Que quede claro. Hablemos de acoso callejero. Universidad San Francisco de Quito USFQ. http://repositorio.usfq.edu.ec/ bitstream/23000/6368/1/130790.pdf

Rodríguez, P. (2020). Acoso sexual callejero: un análisis criminológico y con perspectiva de género. Tesis de Grado. Universidad Autónoma de Barcelona, Barcelona. https://ddd.uab.cat/ record/229050

Stop Street Harassment. (2014). What Is Street Harassment? http://stopstreetharassment.org/ about/what-is-street-harassment/ 